\title{
Grain Boundary Engineering at the Interface of Ceramic and Composite Materials used in Alternative Energy Technologies
}

\author{
L. Saraf ${ }^{1}$, T. Darroudi ${ }^{1}$, O. Dillon ${ }^{1}$, J. J. Clarke ${ }^{2}$ and G. Wetzel $^{1}$ \\ ${ }^{1 .}$ Electron Microscopy Laboratory, Clemson University, Clemson SC 29634 \\ 2. Hitachi High Technologies America Inc., Clarksburg, MD 20871
}

Complex ceramic and composite materials used in alternative energy technologies often present challenges during electron microscopy analysis. Bulk electrical properties such as transport and mobility are crucial factors in carbon based or nanomaterial composites often analyzed at the atomic or molecular level despite the presence of localized chemical composition and resultant phase transformation along its interface. Such materials often undergo transformations in dynamic environments such as a combination of high temperature and reducing gases. Data derived from multiple electron microscopy analytical techniques are presented as a focus for grain boundary engineering in ceramic and composite materials. The materials are used in solid oxide fuel cells and Li-ion batteries for applications related to the development of catalysts and novel ion conducting materials for alternative energy technologies.

Despite the potential of low cost and high theoretical energy density, large capacity degradation occurs in lithium-sulfur batteries. A recent report of a new design of lithium-sulfur batteries using electrically connected graphite and lithium metal as a hybrid anode is used to control undesirable surface reactions on lithium [1]. Sulfur chemical mapping in combination with electrochemical testing revealed significant improvements in battery performance. Recent analysis of nanocellular carbon foam indicated that a functionalized surface area leads to better sodium ion insertion and energy storage [2]. Better sodium ion insertion is recently attributed to short diffusion distance in the hollow carbon nanowires [3]. Similarly, self-assembled $\mathrm{TiO}_{2}$-graphene hybrid nanostructures facilitate enhanced Li-ion insertion [4]. All of these studies indicate the importance of surface and interface engineering to improve energy storage. Electron microscopy analysis plays a crucial part during the design of novel composite materials.

In Solid Oxide Fuel Cell (SOFC), electricity is electrochemically generated using oxygen ion mobility from oxidizing fuel at high temperature. Our past analysis of materials used in SOFC indicates importance of grain boundaries and interfaces to achieve high performance in the fuel cells [5-7]. Grain boundary engineering is important to achieve the desired Triple Phase Boundary (TPB) area at the anode/electrolyte and cathode/electrolyte interface. Typically, nanomaterials like $\mathrm{CeO}_{2}$ along with $\mathrm{NiO}$ play an important role along the anode/electrolyte interface due to its high catalytic activity as a result of high surface area. The images in Figure 1 (a-e) show (EBSD) phase map, (EDX) oxygen Ka map, SEM, (EDX) cerium La line map and (EBSD) inverse pole figure map, respectively at the interface between anode/electrolyte. Dense fluorite electrolyte in SOFC is important to avoid direct gas mixing between reducing and oxidizing gases. Porous anode with a combination of $\mathrm{NiO}$ and fluorite ceria structure facilitates a mixed ionic and electronic conduction. Graded porosity with grain boundary engineering is created at the interface to achieve high TPB area. The darker band in IPF analysis at the interface is a non-indexed region due to the presence of nanocrystalline ceria at the interface. As shown in the Figure 1(b) and 1(d), ceria at the interface is confirmed using the EDX mapping from $\mathrm{Ce} \mathrm{La}$ and $\mathrm{O} \mathrm{Ka}$ lines. Similar results are obtained at the cathode/electrolyte interface. The 3-D analysis at both interfaces also confirmed graded porosity. EM analysis using such ceramic oxides and composite materials will be 
discussed that underscores the importance of grain boundary engineering at the electrode interfaces in alternative energy technology research.

\section{References}

[1] C Huang, J Xiao, Y Shao, J Zheng, W Bennett, D Lu, L Saraf, M Engelhard, L Ji, J Zhang, X Li, G Graff, J Liu, Nature Communications, 5 (2014), 3015.

[2] Y Shao, J Xiao, W Wang, M Engelhard, X Chen, Z Nie, M Gu, L Saraf, G Exarhos, J Zhang, J Liu, Nano Letters, 13 (2013), 3909.

[3] Y Cao, L Xiao, M Sushko, W Wang, B Schwenzer, J Xiao, Z Nie, L Saraf, Z Yang, J Liu, Nano Letters, 12 (2012), 3783.

[4] D Wang, D Choi, J Li, Z Yang, Z Nie, R Kou, D Hu, C Wang, L Saraf, J Zhang, I Aksay, J Liu, ACS Nano, 3 (2009), 907.

[5] L Saraf, Microscopy and Microanalysis, 18 (2012), 371

[6] L Saraf, D Baer, A Lea, Z Zhu, J Strohm, S Sitzman, D King, Journal of the Electrochemical Society, 157 (2010), B463.

[7] L Saraf, V Shutthanandan, Y Zhang, S Thevuthasan, C Wang, A El-Azab, D Baer, Journal of Applied Physics, 96 (2004), 5756.

[8] The authors acknowledge usage of microscopy resources at Clemson University, Hitachi High Technologies America and at Pacific Northwest National Laboratory for this work.

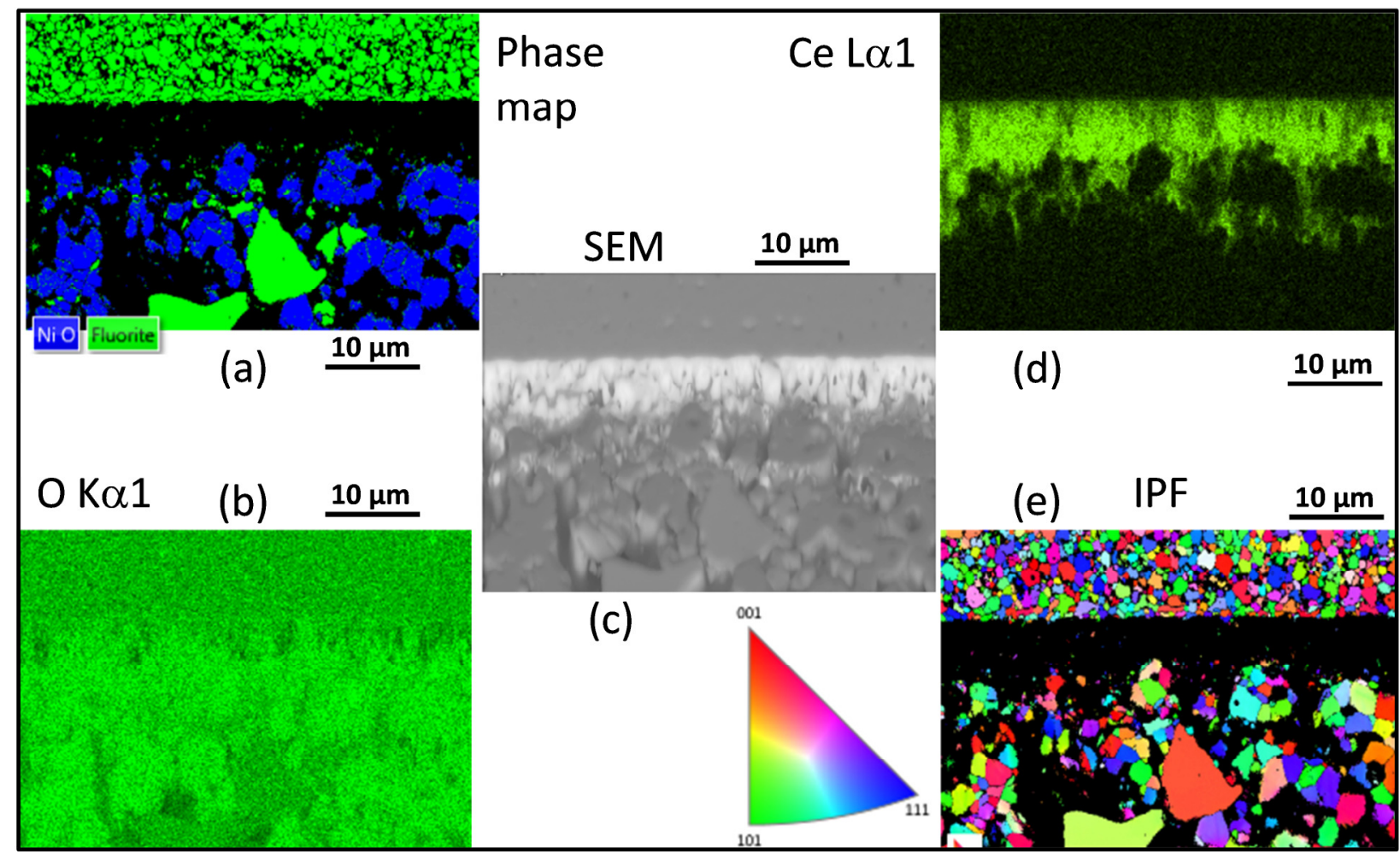

Figure 1 - EDX and EBSD analysis of SOFC stack at the anode/electrolyte interface. Set of images in (a-e) show (EBSD) phase map, (EDX) oxygen K map, SEM, (EDX) cerium L map and (EBSD) inverse pole figure map, respectively. 